\title{
Experimental programme on absolute fission fragment yields with the Lohengrin spectrometer: New optical and statistical methodologies
}

\author{
Chebboubi Abdelaziz ${ }^{1,2}$, Kessedjian Grégoire ${ }^{1, \text { a }}$, Serot Olivier $^{2}$, Julien-Laferriere Sylvain ${ }^{1,2}$, Sage Christophe ${ }^{1}$, \\ Martin Florence ${ }^{1}$, Méplan Olivier ${ }^{1}$, Bernard David ${ }^{2}$, Litaize Olivier ${ }^{2}$, Blanc Aurélien ${ }^{3}$, Faust Herbert ${ }^{3}$, Mutti Paolo ${ }^{3}$, \\ Köster Ulli ${ }^{3}$, Letourneau Alain ${ }^{4}$, Materna Thomas ${ }^{4}$, and Rapala Michal ${ }^{4}$ \\ ${ }^{1}$ LPSC, Université Grenoble-Alpes, CNRS/IN2P3, 53 rue des Martyrs, 38026 Grenoble Cedex, France \\ 2 CEA, DEN, DER, SPRC, LEPh, Cadarache center, 13108 Saint-Paul-lez-Durance, France \\ 3 Institut Laue-Langevin, 38042 Grenoble Cedex 9, France \\ ${ }^{4}$ CEA, DSM, IRFU, SPhN, LEARN, Saclay center, 91191 Gif-sur-Yvette, France
}

\begin{abstract}
The study of fission yields has a major impact on the characterization and understanding of the fission process and is mandatory for reactor applications. In the past with the LOHENGRIN spectrometer of the ILL, priority has been given for the studies in the light fission fragment mass range. The LPSC in collaboration with ILL and CEA has developed a measurement program on symmetric and heavy mass fission fragment distributions. The combination of measurements with ionisation chamber and Ge detectors is necessary to describe precisely the heavy fission fragment region in mass and charge. Recently, new measurements of fission yields and kinetic energy distributions are has been made on the ${ }^{233} U\left(n_{t h}, f\right)$ reaction. The focus of this work has been on the new optical and statistical methodology and the self-normalization of the data to provide new absolute measurements, independently of any libraries, and the associated experimental covariance matrix.
\end{abstract}

\section{Nuclear data}

The accurate knowledge of fission data in the actinide region is important for studies of innovative nuclear reactor concepts. Today, in the framework of nuclear data evaluation, fission models are necessary to increase the consistency and the precision of the libraries. For instance, post neutron fission yields are actually needed in the current and innovative fuel cycles for the calculation of the inventory and the radiotoxicity of the spent fuel, and the estimation of the residual power after shutdown.

Moreover, fission yield measurements supply experimental data to put constraints on fission models and improve their predictive power. In this context, since 2007, various experiments from our collaboration have been performed to investigate fission yields at the Lohengrin spectrometer of the ILL with a special focus on the heavy and symmetry mass region, where there are inconsistencies between models (or evaluations) and the few experimental data.

Recent measurements from [1-3] of ${ }^{233} \mathrm{U}$ fission yields in the heavy region with the Lohengrin spectrometer showed an important discrepancy near the symmetry region in comparison with the evaluations. Thus a new methodology has been developed $[3,4]$ to disentangle the dispersion of measurements in the heavy peak mass region in the descent to symmetrical masses.

\section{Experimental setup}

The Lohengrin mass spectrometer [4] is a nuclear physics instrument of the ILL research reactor facility which allows the study of fragment distributions from thermal neutron induced fission with a very good resolution. A fissile actinide target is placed close to the reactor core, in a thermal neutron flux reaching $5 \times 10^{14}$ neutron. $\mathrm{cm}^{-2} \cdot \mathrm{s}^{-1}$.

Fission fragments emerge from the target with an ionic charge distributed around an average ionic charge of about 21 . Those fragments are emitted along the beam tube axis undergo a horizontal deflection in a magnetic field, directly followed by a vertical deflection in an electric field.

These combined fields separate ions according to their $\mathrm{A} / \mathrm{q}$ and $\mathrm{E} / \mathrm{q}$ ratios, with $\mathrm{A}, \mathrm{q}$ and $\mathrm{E}$ the mass, ionic charge and kinetic energy of the ions respectively. At the spectrometer exit, different detection systems can be installed, such as an ionisation chamber for mass yield measurements, or Ge clovers. Note that the last are used with an additional magnet which aims to focus the ion beam. A schematic view of the spectrometer is shown in Fig. 1.

\section{Relative and absolute experimental mass yields}

\subsection{Expected analysis protocol}

Measurements of fission yields with the Lohengrin spectrometer are in principle simple. Because of the

\footnotetext{
a e-mail: kessedjian@lpsc.in2p3.fr
} 


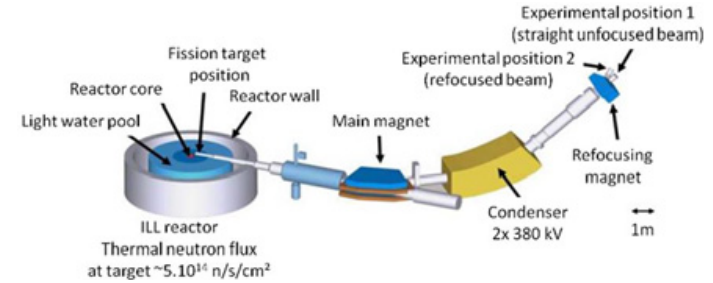

Figure 1. Lohengrin spectrometer scheme. Position 1 is basically dedicated to the mass yield measurement. Position 2 are available for gamma spectrometry using Ge Clover and BEGe detectors.

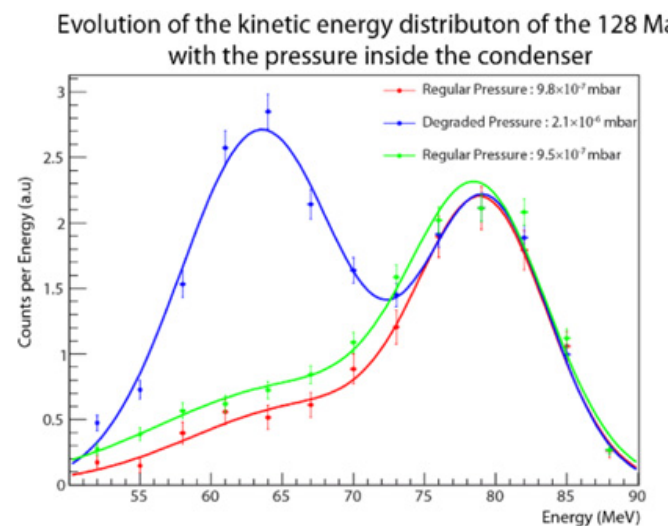

Figure 2. Influence of the condenser pressure on the kinetic energy distribution.

Lohengrin selection, before assessing fission yields, one has to measure the fission rate as function of kinetic energy and ionic charge. However, measurements all over these distributions are so time consuming that choices have to be made to obtain an estimation of the mass or isotopic rates. To limit the bias in the analysis due to the ionic charge - kinetic energy correlation, at least three kinetic energy distributions and one ionic charge distribution are completely measured with a probability lower than $1 \%$.

The second important point concerns the burn-up, corresponding to the evolution of the quantity of actinide in the target. Since the mass 136 is very clean on the Lohengrin spectrometer, each 4 to $6 \mathrm{~h}$, we achieved a fully description of the ionic and kinetic energy distributions of this mass. This point is now coupled to the beam alignment measurement corresponding to a magnetic scan of the main magnet. Thus, with the same frequency, we determine an estimation of the Lohengrin acceptance $(\sim 99 \%)$ to take into account all the systematic uncertainties in the analysis.

If isotopic or mass distributions are covered, it is possible to obtain a self-normalisation of the data and then an absolute measurement of the fission yields. In other cases, a cross normalisation with the nuclear libraries is needed which induces a new systematic uncertainty.

\subsection{Contamination highlighting}

In the descent to the symmetry region $(\mathrm{A}<130)$, a second component appears at low kinetic energy. We previously observed this component to be sensitive to the pressure of the spectrometer. The evolution of this effect with pressure is shown in Fig. 2. This phenomenon is explained as a consequence of ionic charge exchange between the fission fragment and the residual gas of the Lohengrin vacuum [4].

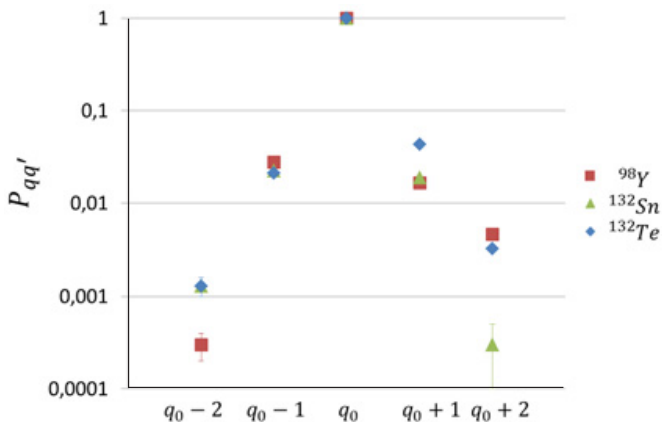

Figure 3. Measurements of the probability of charge changing using gamma spectrometry.

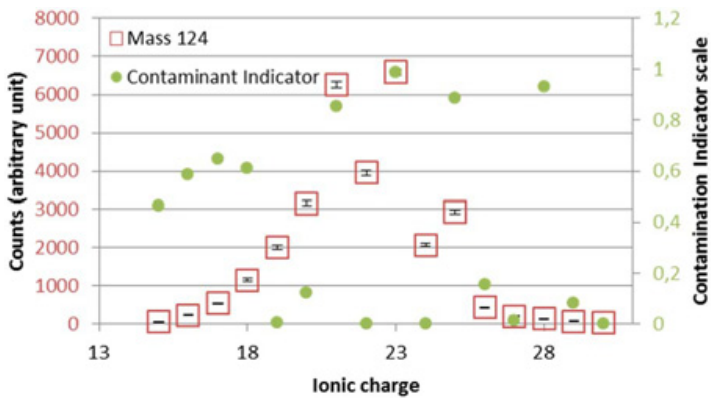

Figure 4. Contamination indicator for the mass 124 as a function of ionic charge.

The validation of this assumption was obtained with an experimental campaign using the gamma spectrometry at the experimental position 2 to identify the unexpected masses. For this, we chose a couple of main magnet settings at two different ionic charge which selects or not the interesting mass. The first Lohengrin measurement corresponds to a normal setting. The second one is achieved using only the setting of the main magnet for the neighboring ionic charge but conserving the first setting for the condenser. Thus we can estimate the probability of charge changing in the lohengrin due to the atomic collision with the residual gas in the spectrometer. Figure 3 presents these results for three isotopes.

Figure 4 shows the indicator of contamination for the ionic charge distribution of the mass 124. The masses with discrepant count rates out of the distribution shaped by the other ones are typically the masses with the highest contamination indicator values. This indicator was validated by gamma spectrometry, where the presence of the expected contaminant was proven.

Figure 5 presents the sketch of the optical operating principle and the real one. Considering this analysis, we have been able to develop a new procedure to analyse the low mass yields perturbed by high mass yields when few percent of rate describes an abnormal trajectory due to the charge changing.

\subsection{Analysis method for the contamination correction}

Equation (1) corresponds to the correction of the expected masse where contaminations are non-negligible.

$$
\begin{aligned}
& P\left(E_{k}\right)^{S y m}=P\left(E_{k}\right)^{\text {mes }} \\
& \quad-\sum_{\text {Contaminant }} P_{q q^{\prime}} \times P_{i m g} \frac{Y\left(A_{\text {cont }}\right)}{Y\left(A_{\text {Sym }}\right)} \times P\left(E_{k}\right)^{\text {cont }}
\end{aligned}
$$




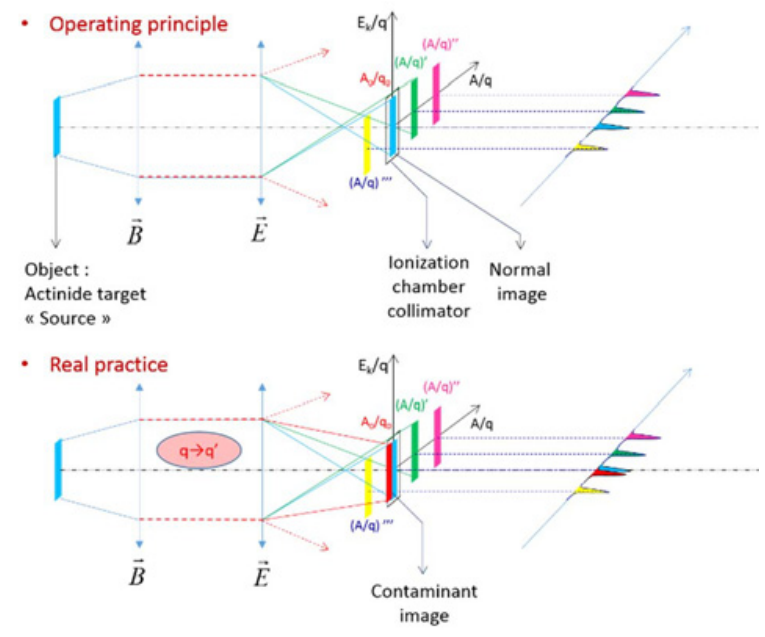

Figure 5. Scheme illustrating the optical analogy of Lohengrin spectrometer principle in order to visualise the origin of the contaminant distribution.

Where $P\left(E_{k}\right)^{\text {Sym }}$ and $P\left(E_{k}\right)^{\text {mes }}$ are the kinetic energy distributions before and after corrections, $P_{q q^{\prime}}$ are the charge changing probability, $\mathrm{Y}$ the mass yields and $P\left(E_{k}\right)^{\text {cont }}$ the measured kinetic energy distribution of the contaminant; $P_{\text {img }}$ describes the part of contaminant image passing through the collimator and corresponding to the contaminant indicator.

Then for each measurement in the symmetry region, we need the ionic charge and kinetic energy distributions of the expected and contaminant masses. Results of different campaign are shown in Fig. 6 where these measurements are displayed with the JEFF-3.1.1 evaluations [5] and the GEF code [6]. The campaigns of F. Martin et al. [1] have described all the heavy peak allowing the self-normalisation of the fission yields. Then this result can be considered as an absolute measurement. The present work deals with the symmetrical masses but a lot of heavy masses correspond to the contaminants. Then we obtain many heavy mass yields to normalise the present results to Ref. [1] and we observe a perfect agreement according to the uncertainties. Present results called "soft correction" correspond to the corrections using Eq. (1). Raw correction data correspond to the maximal subtraction of contaminants considering $P_{q q^{\prime}}$ as free parameters. We observe a disagreement between our results and the evaluation or GEF model which have been interpreted in previous communications [3,4]. This work's ambition is to study the structures in the fission fragment kinetic energy distributions and use symmetric fission as a laboratory to test fission models.

\section{Experimental fission yields covariance}

Nowadays, determination of covariance matrices is one of the most important tasks in order to improve the consistency and precision of nuclear data. In this context, experimental data must provide clear information on systematic and statistical uncertainty to help the evaluation process. The context of new studies of fission yields for nuclear application needs the determination of the covariance matrices. Nevertheless it is necessary to clarify

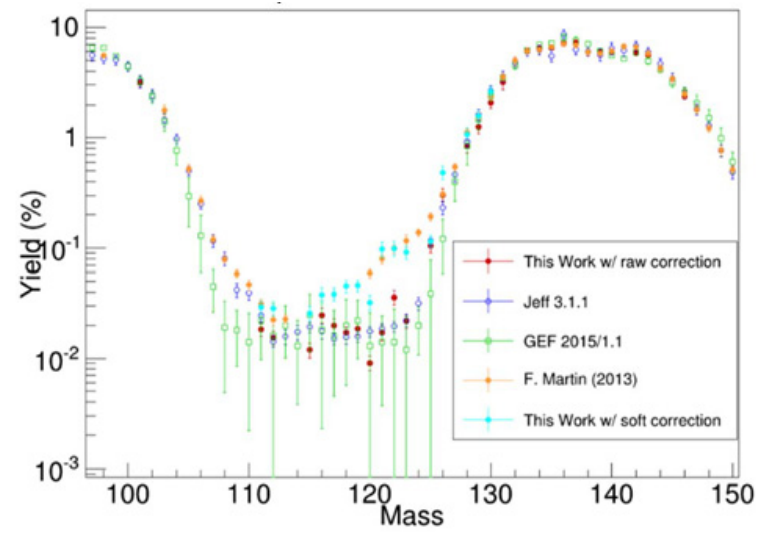

Figure 6. neutron induced ${ }^{233} \mathrm{U}$ mass yields per component in kinetic energy distributions compared to the Jeff-3.1.1 library and the GEF Code.
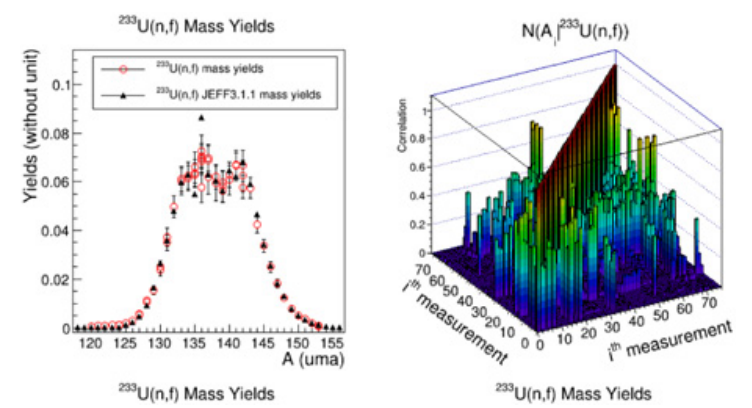

Figure 7. (Left) ${ }^{233} \mathrm{U}\left(\mathrm{n}_{\mathrm{th}}, \mathrm{f}\right)$ Mass yields obtained by F. Martin et al. compared to the JEFF 3.1.1 library. (Right) Experimental covariance matrix of fission rate before normalization.

what are the ingredients of the covariance for the evaluations to consider one of the systematic effects.

\subsection{Experimental mass yield covariance}

With the Lohengrin spectrometer we are only able to measure relative fission rate. Nevertheless, considering the light or heavy mass region or both, it is possible to normalize the fission yields and obtain an absolute measurement without any standard reaction. Different sources of systematic uncertainty can be found on the Lohengrin spectrometer. These are due to burnup correction, incomplete ionic and kinetic energy distributions or the Lohengrin acceptance. By definition two mass yields which are a priori independent, are correlated because of their common uncertainty. Moreover, due to the life-time of the target $(\sim 1$ week), two or three targets are necessary to cover the heavy fission fragment peak. Cross normalisation induces a further new systematic uncertainty, linking the data of the second target to the first one. Figure 7 presents the experimental covariance of our results on ${ }^{233} \mathrm{U}\left(\mathrm{n}_{\mathrm{th}}, \mathrm{f}\right)$ mass yields $[2,7]$.

\subsection{Pure experimental evaluation and covariance}

The normalisation of all mass yields to unity for each peak (or to 2 for both fragments) induces negative correlation since the sum is definitively fixed [6]. Then, even considering only positive correlation in experimental data, a negative component appears in the covariance of 

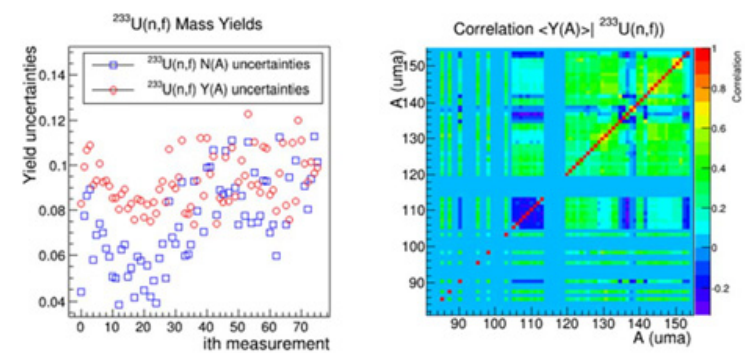

Figure 8. Left: ${ }^{233} \mathrm{U}\left(\mathrm{n}_{\mathrm{th}}, \mathrm{f}\right)$ mass rate $\mathrm{N}(\mathrm{A})$ [1] and mass yield $\mathrm{Y}(\mathrm{A})$ uncertainties compared to the JEFF 3.1.1 library. Right: experimental covariance matrix of fission rate before normalization [7].

the normalized results (see Fig. 7). This normalisation of fission rate N(A) generates mass yields $\mathrm{Y}(\mathrm{A})$ with the following consequences: $\mathrm{i}$ ) an increasing of the $\mathrm{Y}(\mathrm{A})$ uncertainties in comparison to N(A) for the mass A due to the uncertainty contributions of other masses (see Fig. 8 right), ii) but a negative correlation between two different masses. These results correspond to an absolute measurement of mass yields which can be considered as a pure experimental assessment of the fission yields [7].

\subsection{Comparison with other covariance generation methods}

Different methodologies to generate the fission yield covariance have been proposed in the JEFF Group. The basic methodologies are to assume the measurements as independent and to consider only the negative correlation due to the normalisation. This assumption is probably limited since systematic uncertainties often rule total uncertainty. In other words, for a given setup, because of the experimental conditions, all measurements are intrinsically bounded. N. Terranova et al. have developed a code to compare the pre-neutron emission fission fragments to the JEFF library considering the neutron emission per mass, the so-called saw-tooth, as parameters [8]. The good agreement of this observable allows the authors to consider the coherence of the procedure, and then extract the fission yields correlation of the adjustment.

In the same way, the GEF code extracts the fission yield correlation of the covariance of all model parameters allowing a coherence between the descriptions of all fission systems and the cross correlation between the systems. Naturally all these covariance cannot consider the missing experimental covariance of nuclear data library but the present work shows the effects of the systematics on experimental covariance matrix. Thus, the adjusted model parameters of each method could be perturbed by the experimental covariance and then many changes in the final covariance could be expected.

\section{Conclusion and perspectives}

A new analysis procedure has been developed to measure absolute fission yields with the Lohengrin spectrometer. Contaminant correction in the low yield region have been highlighted and corrected to improve the robustness of the results all over the mass range. Covariance terms are due to the self-normalisation but also to the systematic uncertainties from the analysis.

However, only relative measurement, called fission rates, have to be consider to the usual JEFF evaluation process, in order to include once the normalisation uncertainties. To test models or evaluations, absolute experimental data with the associated covariance matrices are requested in order to compare independent models and the proposed absolute data sets.

\section{References}

[1] F. Martin et al., Proc. 2nd ANIMMA Conference, June 2011, Ghent

[2] F. Martin et al., Nucl. Data Sheets 119, 328-330 (2014)

[3] A. Chebboubi, PhD thesis, University GrenobleAlpes, 2015

[4] A. Chebboubi, G. Kessedjian, C. Sage et al. Wonder conference 2015

[5] The JEFF-3.1.1 nuclear data library, OECD, NEA, JEFF Report 21, ISBN 92-64-02314-3 (2006)

[6] K.H. Schmidt et al., Nucl. Data Sheets 131, 107-221 (2016)

[7] G. Kessedjian, HDR degree, Grenoble University, 2015

[8] N. Terranova et al., Nuclear Data Sheets 123, 225-230 (2015) 